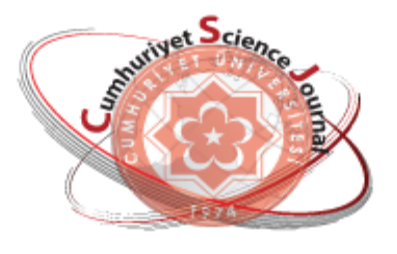

e-ISSN: $2587-246 X$

ISSN: $2587-2680$

\section{Cumbenriy ot Science Journal \\ esg}

Cumhuriyet Sci. J., Vol.40-4 (2019) 886-895

\title{
A Study on First-Order Fuzzy Initial Value Problems with Positive and Negative Fuzzy Number Coefficients by Fuzzy Laplace Transform
}

\author{
Hülya GÜLTEKİN ÇITIL \\ Department of Mathematics, Faculty of Arts and Sciences, Giresun University, Giresun, Turkey.
}

Received: 14.10.2019; Accepted: 21.11.2019

http://dx.doi.org/10.17776/csj.632770

\begin{abstract}
In this paper, fuzzy initial value problems for first-order fuzzy differential equations with positive and negative fuzzy number coefficients are studied by fuzzy Laplace transform. Solutions are found under the approach of generalized differentiability. Examples are solved. Figures of the solutions are drawn using the Mathematica program. Finally, conclusions are given.
\end{abstract}

Keywords: Fuzzy Initial Value Problem, Generalized Differentiability, Fuzzy Laplace Transform, Positive and Negative Fuzzy Numbers.

\section{Fuzzy Laplace Dönüşümüyle Pozitif ve Negatif Fuzzy Sayı Katsayılı Birinci-Mertebe Fuzzy Başlangıç Değer Problemleri Üzerine Bir Çalışma}

Özet. Bu çalışmada, fuzzy Laplace dönüşümüyle pozitif ve negatif fuzzy sayı katsayılı birinci-mertebe fuzzy diferansiyel denklemler için fuzzy başlangıç değer problemleri çalışıldı. Çözümler genelleştirilmiş diferansiyellenebilirlik yaklaşımı altında bulundu. Örnekler çözüldü. Çözümlerin şekilleri Mathematica programı kullanarak çizildi. Son olarak, sonuçlar verildi.

Anahtar Kelimeler Fuzzy Başlangıç Değer Problem, Genelleştirilmiş Diferansiyellenebilirlik, Fuzzy Laplace Dönüşüm, Pozitif ve Negatif Fuzzy Sayllar.

\section{INTRODUCTION}

The topic of fuzzy differential equations has been rapidly grown in current years. The term of "fuzzy differetial equation" was introduced by Kandel and Byatt [1]. The fuzzy differential equations are extensively used in applied mathematics, physics and engineering. Many researchers study fuzzy differential equation with different approach. The first approach is on the Zadeh's extenciple [2]. The second approach is differential inclusion [3]. The third approach is Hukuhara derivative [4-5] or generalized derivative [6-8].

Fuzzy Laplace transform is very useful to solve fuzzy differential equation. In many papers, fuzzy Laplace transform was studied [9-11].

This paper is on solutions of fuzzy initial value problems for first-order fuzzy differential equations with positive and negative fuzzy number coefficients by fuzzy Laplace transform. 
Definition 1.1 [12] A fuzzy number is a function $u: \mathbb{R} \rightarrow[0,1]$ satisfying the following properties:

$u$ is normal, $u$ is convex fuzzy set, $u$ is upper s

emi-continuous on $\mathbb{R}$ and $c l\{x \in \mathbb{R} \mid u(x)>0\}$ is compact, where $c l$ denotes the closure of a subset.

Let $\mathbb{R}_{F}$ be the space of fuzzy numbers.

Definition 1.2 [12] Let be $u \in \mathbb{R}_{F}$. The $\alpha$-level set of $u$ is $[u]^{\alpha}=\{x \in \mathbb{R} \mid u(x) \geq \alpha\}, 0<\alpha \leq 1$.

If $\alpha=0$, the support of $u$ is $[u]^{0}=\operatorname{cl}\{x \in \mathbb{R} \mid u(x)>0\}$.

Definition 1.3 [13] A fuzzy number $u$ in parametric form is a pair $\left[\underline{u}_{\alpha}, \bar{u}_{\alpha}\right]$ of functions $\underline{u}_{\alpha}, \bar{u}_{\alpha}, 0 \leq \alpha \leq 1$, which satisfy the following requirements:

1. $\underline{u}_{\alpha}$ is bounded non-decreasing left-continuous in $(0,1]$, right-continuous at $\alpha=0$.

2. $\bar{u}_{\alpha}$ is bounded non-increasing left-continuous in $(0,1]$, right-continuous at $\alpha=0$.

3. $\underline{u}_{\alpha} \leq \bar{u}_{\alpha}, 0 \leq \alpha \leq 1$.

Definition 1.4 [14] A fuzzy number $u$ is called positive (negative), denoted by $u>0(u<0)$, if its membership function $u(x)$ satisfies $u(x)=0, \forall x<0(x>0)$.

Definition 1.5 [12] If $A$ is a symmetric triangular number with support $[\underline{a}, \bar{a}]$, the $\alpha$-level set of $A$ is

$$
[A]^{\alpha}=\left[\underline{a}+\left(\frac{\bar{a}-\underline{a}}{2}\right) \alpha, \bar{a}-\left(\frac{\bar{a}-\underline{a}}{2}\right) \alpha\right] .
$$

Definition 1.6 [15] Let be $u, v \in \mathbb{R}_{F},[u]^{\alpha}=\left[\underline{u}_{\alpha}, \bar{u}_{\alpha}\right],[v]^{\alpha}=\left[\underline{v}_{\alpha}, \bar{v}_{\alpha}\right]$, the product $u . v$ is defined by

$$
[u . v]^{\alpha}=[u]^{\alpha} \cdot[v]^{\alpha}, \forall \alpha \in[0,1]
$$

where

$$
\begin{gathered}
{[u]^{\alpha} \cdot[v]^{\alpha}=\left[\underline{u}_{\alpha}, \bar{u}_{\alpha}\right]\left[\underline{v}_{\alpha}, \bar{v}_{\alpha}\right]=\left[\underline{w}_{\alpha}, \bar{w}_{\alpha}\right],} \\
\underline{w}_{\alpha}=\min \left\{\underline{u}_{\alpha} \underline{v}_{\alpha}, \underline{u}_{\alpha} \bar{v}_{\alpha}, \bar{u}_{\alpha} \underline{v}_{\alpha}, \bar{u}_{\alpha} \bar{v}_{\alpha}\right\}, \bar{w}_{\alpha}=\max \left\{\underline{u}_{\alpha} \underline{v}_{\alpha}, \underline{u}_{\alpha} \bar{v}_{\alpha}, \bar{u}_{\alpha} \underline{v}_{\alpha}, \bar{u}_{\alpha} \bar{v}_{\alpha}\right\} .
\end{gathered}
$$

Definition 1.7 [16] Let be $u, v \in \mathbb{R}_{F}$. If there exists $w \in \mathbb{R}_{F}$ such that $u=v+w$ then $w$ is called the Hdifference of $u$ and $v$ and it is denoted $u \ominus v$.

Definition 1.8 [16] Let be $f:[a, b] \rightarrow \mathbb{R}_{F}$ and $t_{0} \in[a, b]$. If there exists $f^{\prime}\left(t_{0}\right) \in \mathbb{R}_{F}$ such that for all $h>0$ sufficiently small, $\exists f\left(t_{0}+h\right) \ominus f\left(t_{0}\right), f\left(t_{0}\right) \ominus f\left(t_{0}-h\right)$ and the limits hold

$$
\lim _{h \rightarrow 0} \frac{f\left(t_{0}+h\right) \ominus f\left(t_{0}\right)}{h}=\lim _{h \rightarrow 0} \frac{f\left(t_{0}\right) \ominus f\left(t_{0}-h\right)}{h}=f^{\prime}\left(t_{0}\right),
$$

$f$ is (1)-differentiable at $t_{0}$. If there exists $f^{\prime}\left(t_{0}\right) \in \mathbb{R}_{F}$ such that for all $h>0$ sufficiently small, $\exists f\left(t_{0}\right) \ominus$ $f\left(t_{0}+h\right), f\left(t_{0}-h\right) \ominus f\left(t_{0}\right)$ and the limits hold

$$
\lim _{h \rightarrow 0} \frac{f\left(t_{0}\right) \ominus f\left(t_{0}+h\right)}{-h}=\lim _{h \rightarrow 0} \frac{f\left(t_{0}-h\right) \ominus f\left(t_{0}\right)}{-h}=f^{\prime}\left(t_{0}\right),
$$

$f$ is (2)-differentiable.

Definition 1.9 [13] The fuzzy Laplace transform of fuzzy function $f$ is 


$$
\begin{aligned}
& F(s)=L(f(t))=\int_{0}^{\infty} e^{-s t} f(t) d t=\left[\lim _{\rho \rightarrow \infty} \int_{0}^{\rho} e^{-s t} \underline{f}(t) d t, \lim _{\rho \rightarrow \infty} \int_{0}^{\rho} e^{-s t} \bar{f}(t) d t\right] . \\
& F(s, \alpha)=L\left([f(t)]^{\alpha}\right)=\left[L\left(\underline{f}_{\alpha}(t)\right), L\left(\bar{f}_{\alpha}(t)\right)\right], \\
& L\left(\underline{f_{\alpha}}(t)\right)=\int_{0}^{\infty} e^{-s t} \underline{f_{\alpha}}(t) d t=\lim _{\rho \rightarrow \infty} \int_{0}^{\rho} e^{-s t} \underline{f_{\alpha}}(t) d t \\
& L\left(\bar{f}_{\alpha}(t)\right)=\int_{0}^{\infty} e^{-s t} \bar{f}_{\alpha}(t) d t=\lim _{\rho \rightarrow \infty} \int_{0}^{\rho} e^{-s t} \bar{f}_{\alpha}(t) d t .
\end{aligned}
$$

Theorem 1.1 [9] Let $f^{\prime}(t)$ be an integrable fuzzy function and $f(t)$ is primitive of $f^{\prime}(t)$ on $(0, \infty]$.

1. If $f$ is (1)-differentiable, $L\left(f^{\prime}(t)\right)=s L(f(t)) \ominus f(0)$.

2. If $f$ is (2)-differentiable, $L\left(f^{\prime}(t)\right)=(-f(0)) \ominus(-s L(f(t)))$.

\section{RESULTS AND DISCUSSION}

Consider the fuzzy problem

$$
\begin{gathered}
u^{\prime}(t)+[\mu]^{\alpha} u(t)=[\rho]^{\alpha}, t>0 \\
\mathrm{u}(0)=[\omega]^{\alpha},
\end{gathered}
$$

by the fuzzy Laplace transform, where $[\mu]^{\alpha}=\left[\underline{\mu}_{\alpha}, \bar{\mu}_{\alpha}\right],[\rho]^{\alpha}=\left[\underline{\rho}_{\alpha}, \bar{\rho}_{\alpha}\right],[\omega]^{\alpha}=\left[\underline{\omega}_{\alpha}, \bar{\omega}_{\alpha}\right]$ are symmetric triangular fuzzy numbers and $u(t)$ is positive fuzzy function. In this paper, (i) solution means that $u$ is (i)-differentiable, $\mathrm{i}=1,2$.

\section{I) The case of positive fuzzy number coefficient}

Let be $[\mu]^{\alpha}=\left[\underline{\mu}_{\alpha}, \bar{\mu}_{\alpha}\right]$ positive fuzzy number.

1) Let be $u$ (1)-differentiable. Then, using the fuzzy Laplace transform, from the fuzzy differential equation (1)

$$
s L(u(t)) \ominus u(0)+[\mu]^{\alpha} L(u(t))=L\left([\rho]^{\alpha}\right)
$$

is obtained. Using the Hukuhara difference and fuzzy arithmetic, we have

$$
\begin{aligned}
& s L\left(\underline{u}_{\alpha}(t)\right)-\underline{u}_{\alpha}(0)+\underline{\mu}_{\alpha} L\left(\underline{u}_{\alpha}(t)\right)=L\left(\underline{\rho}_{\alpha}\right), \\
& s L\left(\bar{u}_{\alpha}(t)\right)-s \bar{u}_{\alpha}(0)+\bar{\mu}_{\alpha} L\left(\bar{u}_{\alpha}(t)\right)=L\left(\bar{\rho}_{\alpha}\right) .
\end{aligned}
$$

Also, using the initial condition (2), we obtain

$$
\begin{aligned}
& L\left(\underline{u}_{\alpha}(t)\right)=\frac{\underline{\rho}_{\alpha}}{s\left(s+\underline{\mu}_{\alpha}\right)}+\frac{\underline{\omega}_{\alpha}}{s+\underline{\mu}_{\alpha}} \\
& L\left(\bar{u}_{\alpha}(t)\right)=\frac{\bar{\rho}_{\alpha}}{s\left(s+\bar{\mu}_{\alpha}\right)}+\frac{\bar{\omega}_{\alpha}}{s+\bar{\mu}_{\alpha}} .
\end{aligned}
$$


Taking the inverse Laplace transform of this equations, (1) solution is obtained as

$$
\begin{gathered}
\underline{u}_{\alpha}(t)=e^{-\underline{\mu}_{\alpha} t}\left(\underline{\omega}_{\alpha}-\frac{\underline{\rho}_{\alpha}}{\underline{\mu}_{\alpha}}\right)+\frac{\underline{\rho}_{\alpha}}{\underline{\mu}_{\alpha}}, \\
\bar{u}_{\alpha}(t)=e^{-\bar{\mu}_{\alpha} t}\left(\bar{\omega}_{\alpha}-\frac{\bar{\rho}_{\alpha}}{\bar{\mu}_{\alpha}}\right)+\frac{\bar{\rho}_{\alpha}}{\bar{\mu}_{\alpha}} \\
{[u(t)]^{\alpha}=\left[\underline{u}_{\alpha}(t), \bar{u}_{\alpha}(t)\right] .}
\end{gathered}
$$

2) Let be $u$ (2)-differentiable. Using the fuzzy Laplace transform, from the fuzzy differential equation (1)

$$
-u(0) \ominus(-s L(u(t)))+[\mu]^{\alpha} L(u(t))=L\left([\rho]^{\alpha}\right),
$$

that is, the equations

$$
\begin{aligned}
& -\bar{u}_{\alpha}(0)+s L\left(\bar{u}_{\alpha}(t)\right)+\underline{\mu}_{\alpha} L\left(\underline{u}_{\alpha}(t)\right)=L\left(\underline{\rho}_{\alpha}\right), \\
& -\underline{u}_{\alpha}(0)+s L\left(\underline{u}_{\alpha}(t)\right)+\bar{\mu}_{\alpha} L\left(\bar{u}_{\alpha}(t)\right)=L\left(\bar{\rho}_{\alpha}\right)
\end{aligned}
$$

are obtained. From this, we have

$$
\begin{aligned}
& s L\left(\bar{u}_{\alpha}(t)\right)+\underline{\mu}_{\alpha} L\left(\underline{u}_{\alpha}(t)\right)=\frac{\rho_{\alpha}}{s}+\bar{\omega}_{\alpha} \\
& s L\left(\underline{u}_{\alpha}(t)\right)+\bar{\mu}_{\alpha} L\left(\bar{u}_{\alpha}(t)\right)=\frac{\bar{\rho}_{\alpha}}{s}+\underline{\omega}_{\alpha} .
\end{aligned}
$$

If $L\left(\bar{u}_{\alpha}(t)\right)$ in the equation (4) is replaced by the equation (3),

$$
L\left(\underline{u}_{\alpha}(t)\right)=\left(\bar{\rho}_{\alpha}-\bar{\mu}_{\alpha} \bar{\omega}_{\alpha}\right)\left(\frac{1}{s^{2}-\underline{\mu}_{\alpha} \bar{\mu}_{\alpha}}\right)+\underline{\omega}_{\alpha}\left(\frac{s}{s^{2}-\underline{\mu}_{\alpha} \bar{\mu}_{\alpha}}\right)-\frac{\underline{\rho}_{\alpha} \bar{\mu}_{\alpha}}{s\left(s^{2}-\underline{\mu}_{\alpha} \bar{\mu}_{\alpha}\right)}
$$

is obtained. Taking the inverse Laplace transform of this equation, the lower solution is obtained as

$$
\underline{u}_{\alpha}(t)=\frac{\left(\bar{\rho}_{\alpha}-\bar{\mu}_{\alpha} \bar{\omega}_{\alpha}\right)}{\sqrt{\underline{\mu}_{\alpha} \bar{\mu}_{\alpha}}} \sin \left(\sqrt{\underline{\mu}_{\alpha} \bar{\mu}_{\alpha}} t\right)+\underline{\omega}_{\alpha} \cos \left(\sqrt{\underline{\mu}_{\alpha} \bar{\mu}_{\alpha}} t\right)-\frac{\underline{\rho}_{\alpha}}{\underline{\mu}_{\alpha}}\left(\frac{e^{\sqrt{\underline{\mu}_{\alpha} \bar{\mu}_{\alpha}} t}+e^{-\sqrt{\underline{\mu}_{\alpha} \bar{\mu}_{\alpha}} t}}{2}-1\right) .
$$

Similarly, the upper solution is obtained as

$$
\bar{u}_{\alpha}(t)=\frac{\left(\underline{\rho}_{\alpha}-\underline{\mu}_{\alpha} \underline{\omega}_{\alpha}\right)}{\sqrt{\underline{\mu}_{\alpha} \bar{\mu}_{\alpha}}} \sin \left(\sqrt{\underline{\mu}_{\alpha} \bar{\mu}_{\alpha} t}\right)+\bar{\omega}_{\alpha} \cos \left(\sqrt{\underline{\mu}_{\alpha} \bar{\mu}_{\alpha}} t\right)-\frac{\bar{\rho}_{\alpha}}{\bar{\mu}_{\alpha}}\left(\frac{e^{\sqrt{\underline{\mu}_{\alpha} \bar{\mu}_{\alpha} t}}+e^{-\sqrt{\underline{\mu}_{\alpha} \bar{\mu}_{\alpha} t}}}{2}-1\right) .
$$

Example 2.1 Consider the fuzzy initial value problem

$$
\begin{gathered}
u^{\prime}(t)+[1]^{\alpha} u(t)=[2]^{\alpha}, t>0 \\
\mathrm{u}(0)=[1]^{\alpha},
\end{gathered}
$$

using the fuzzy Laplace transform, where $[1]^{\alpha}=[\alpha, 2-\alpha]$, [2] $]^{\alpha}=[1+\alpha, 3-\alpha]$. 
If $u$ is (1)-differentiable, (1) solution is

$$
\begin{gathered}
\underline{u}_{\alpha}(t)=e^{-\alpha t}\left(\alpha-\left(\frac{1+\alpha}{\alpha}\right)\right)+\left(\frac{1+\alpha}{\alpha}\right), \\
\bar{u}_{\alpha}(t)=e^{-(2-\alpha) t}\left((2-\alpha)-\left(\frac{3-\alpha}{2-\alpha}\right)\right)+\left(\frac{3-\alpha}{2-\alpha}\right), \\
{[u(t)]^{\alpha}=\left[\underline{u}_{\alpha}(t), \bar{u}_{\alpha}(t)\right] .}
\end{gathered}
$$

If $u$ is (2)-differentiable, (2) solution is

$$
\begin{gathered}
\underline{u}_{\alpha}(t)=\left(\frac{(3-\alpha)-(2-\alpha)^{2}}{\sqrt{\alpha(2-\alpha)}}\right) \sin (\sqrt{\alpha(2-\alpha)} t)+\alpha \cos (\sqrt{\alpha(2-\alpha)} t)-\left(\frac{1+\alpha}{\alpha}\right)\left(\frac{e^{\sqrt{\alpha(2-\alpha)} t}+e^{-\sqrt{\alpha(2-\alpha)} t}}{2}-1\right), \\
\bar{u}_{\alpha}(t)=\left(\frac{(1+\alpha)-\alpha^{2}}{\sqrt{\alpha(2-\alpha)}}\right) \sin (\sqrt{\alpha(2-\alpha)} t)+(2-\alpha) \cos (\sqrt{\alpha(2-\alpha)} t) \\
-\left(\frac{3-\alpha}{2-\alpha}\right)\left(\frac{e^{\sqrt{\alpha(2-\alpha)} t}+e^{-\sqrt{\alpha(2-\alpha)} t}}{2}-1\right), \\
{[u(t)]^{\alpha}=\left[\underline{u}_{\alpha}(t), \bar{u}_{\alpha}(t)\right] .}
\end{gathered}
$$

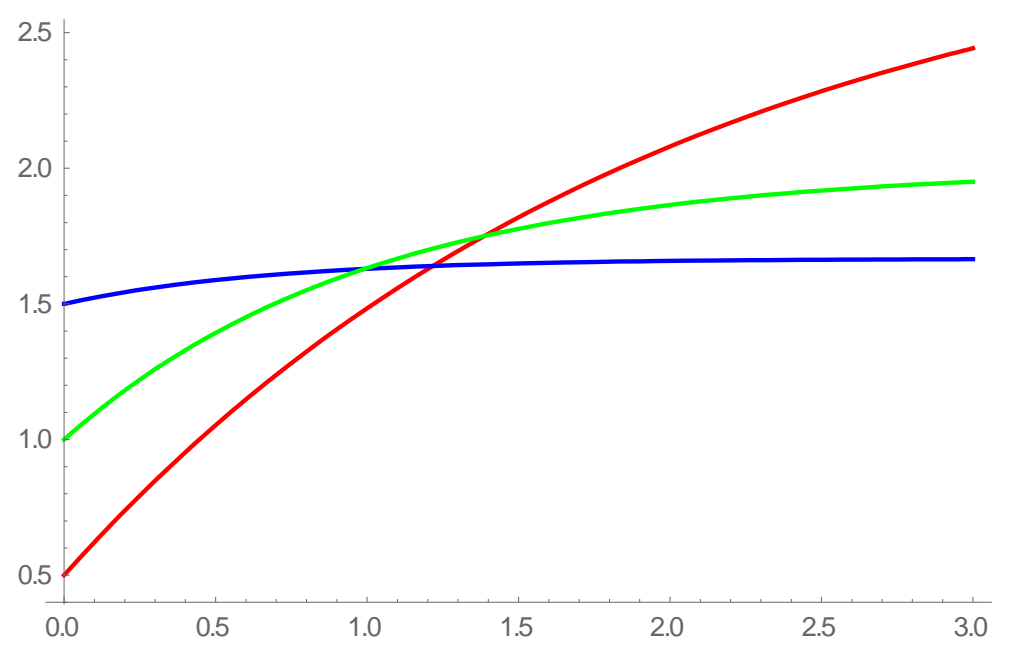

Figure 1 (1) solution for $\alpha=0.5$ 


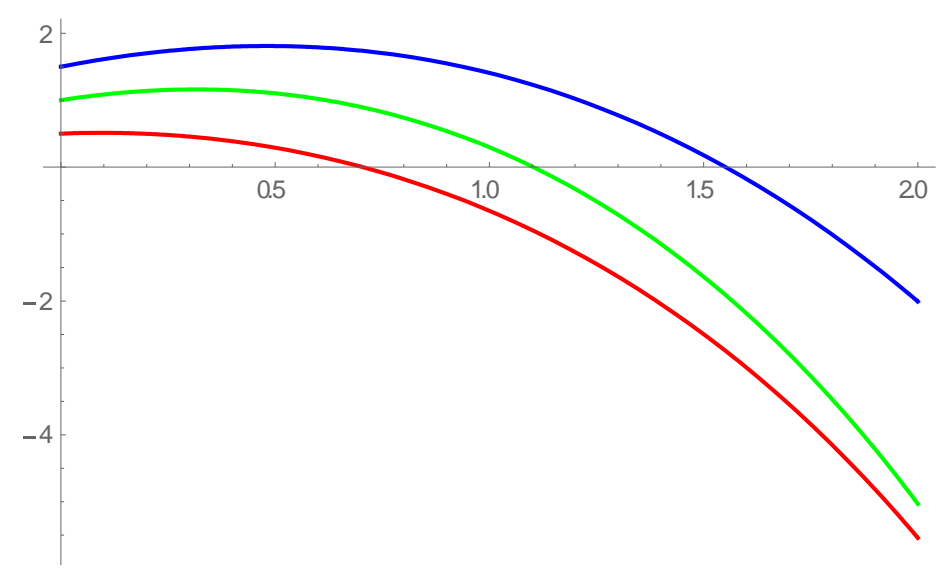

Figure 2 (2) solution for $\alpha=0.5$

$$
\text { red } \rightarrow \underline{u}_{\alpha}(t), \text { blue } \rightarrow \bar{u}_{\alpha}(t), \text { green } \rightarrow u(t)
$$

From Definition 1.3 and since $[u(x)]^{\alpha}$ is positive fuzzy function, according to figure $1,(1)$ solution is a valid fuzzy number for $x \in[0,1.21735]$, according to figure 2 , (2) solution is a valid fuzzy number for $x \in[0,0.704091]$.

\section{II) The case of negative fuzzy number coefficient}

Let be $[\mu]^{\alpha}=\left[\underline{\mu}_{\alpha}, \bar{\mu}_{\alpha}\right]$ negative fuzzy number.

1) Let be $u$ (1)-differentiable. Then, using the fuzzy Laplace transform, the fuzzy differential equation (1) yields

$$
s L(u(t)) \ominus u(0)+[\mu]^{\alpha} L(u(t))=L\left([\rho]^{\alpha}\right) .
$$

From this, using the fuzzy arithmetic and Hukuhara difference

$$
\begin{aligned}
& s L\left(\underline{u}_{\alpha}(t)\right)-\underline{u}_{\alpha}(0)+\underline{\mu}_{\alpha} L\left(\bar{u}_{\alpha}(t)\right)=L\left(\underline{\rho}_{\alpha}\right), \\
& s L\left(\bar{u}_{\alpha}(t)\right)-s \bar{u}_{\alpha}(0)+\bar{\mu}_{\alpha} L\left(\underline{u}_{\alpha}(t)\right)=L\left(\bar{\rho}_{\alpha}\right)
\end{aligned}
$$

are obtained. That is, we have the equations

$$
\begin{aligned}
& s L\left(\underline{u}_{\alpha}(t)\right)+\underline{\mu}_{\alpha} L\left(\bar{u}_{\alpha}(t)\right)=\frac{\rho_{\alpha}}{s}+\underline{\omega}_{\alpha} . \\
& s L\left(\bar{u}_{\alpha}(t)\right)+\bar{\mu}_{\alpha} L\left(\underline{u}_{\alpha}(t)\right)=\frac{\bar{\rho}_{\alpha}}{s}+\bar{\omega}_{\alpha} .
\end{aligned}
$$

If $L\left(\bar{u}_{\alpha}(t)\right)$ in the equation (7) is replaced by the equation (8),

$$
L\left(\underline{u}_{\alpha}(t)\right)=\left(\underline{\rho}_{\alpha}-\underline{\mu}_{\alpha} \bar{\omega}_{\alpha}\right)\left(\frac{1}{s^{2}-\underline{\mu}_{\alpha} \bar{\mu}_{\alpha}}\right)+\underline{\omega}_{\alpha}\left(\frac{s}{s^{2}-\underline{\mu}_{\alpha} \bar{\mu}_{\alpha}}\right)-\frac{\bar{\rho}_{\alpha} \underline{\mu}_{\alpha}}{s\left(s^{2}-\underline{\mu}_{\alpha} \bar{\mu}_{\alpha}\right)}
$$

is obtained. Then, the lower solution is 


$$
\underline{u}_{\alpha}(t)=\frac{\left(\underline{\rho}_{\alpha}-\underline{\mu}_{\alpha} \bar{\omega}_{\alpha}\right)}{\sqrt{\underline{\mu}_{\alpha} \bar{\mu}_{\alpha}}} \sin \left(\sqrt{\underline{\mu}_{\alpha} \bar{\mu}_{\alpha} t}\right)+\underline{\omega}_{\alpha} \cos \left(\sqrt{\underline{\mu}_{\alpha} \bar{\mu}_{\alpha}} t\right)-\frac{\bar{\rho}_{\alpha}}{\bar{\mu}_{\alpha}}\left(\frac{e^{\sqrt{\underline{\mu}_{\alpha} \bar{\mu}_{\alpha}} t}+e^{-\sqrt{\underline{\mu}_{\alpha} \bar{\mu}_{\alpha}} t}}{2}-1\right) .
$$

Similarly, upper solution is

$$
\bar{u}_{\alpha}(t)=\frac{\left(\bar{\rho}_{\alpha}-\bar{\mu}_{\alpha} \underline{\omega}_{\alpha}\right)}{\sqrt{\underline{\mu}_{\alpha} \bar{\mu}_{\alpha}}} \sin \left(\sqrt{\underline{\mu}_{\alpha} \bar{\mu}_{\alpha}} t\right)+\bar{\omega}_{\alpha} \cos \left(\sqrt{\underline{\mu}_{\alpha} \bar{\mu}_{\alpha}} t\right)-\frac{\underline{\rho}_{\alpha}}{\underline{\mu}_{\alpha}}\left(\frac{e^{\sqrt{\underline{\mu}_{\alpha} \bar{\mu}_{\alpha}} t}+e^{-\sqrt{\underline{\mu}_{\alpha} \bar{\mu}_{\alpha}} t}}{2}-1\right) .
$$

2) Let be $u$ (2)-differentiable. Then, using the fuzzy Laplace transform, from the fuzzy differential equation (1)

$$
-u(0) \ominus(-s L(u(t)))+[\mu]^{\alpha} L(u(t))=L\left([\rho]^{\alpha}\right),
$$

that is,

$$
\begin{aligned}
& -\bar{u}_{\alpha}(0)+s L\left(\bar{u}_{\alpha}(t)\right)+\underline{\mu}_{\alpha} L\left(\bar{u}_{\alpha}(t)\right)=L\left(\underline{\rho}_{\alpha}\right), \\
& -\underline{u}_{\alpha}(0)+s L\left(\underline{u}_{\alpha}(t)\right)+\bar{\mu}_{\alpha} L\left(\underline{u}_{\alpha}(t)\right)=L\left(\bar{\rho}_{\alpha}\right)
\end{aligned}
$$

are obtained. From this, we have

$$
\begin{aligned}
& L\left(\underline{u}_{\alpha}(t)\right)=\frac{\bar{\rho}_{\alpha}}{s\left(s+\bar{\mu}_{\alpha}\right)}+\frac{\underline{\omega}_{\alpha}}{s+\bar{\mu}_{\alpha}}, \\
& L\left(\bar{u}_{\alpha}(t)\right)=\frac{\underline{\rho}_{\alpha}}{s\left(s+\underline{\mu}_{\alpha}\right)}+\frac{\bar{\omega}_{\alpha}}{s+\underline{\mu}_{\alpha}} .
\end{aligned}
$$

Then, (2) solution is obtained as

$$
\begin{gathered}
\underline{u}_{\alpha}(t)=e^{-\bar{\mu}_{\alpha} t}\left(\underline{\omega}_{\alpha}-\frac{\bar{\rho}_{\alpha}}{\bar{\mu}_{\alpha}}\right)+\frac{\bar{\rho}_{\alpha}}{\bar{\mu}_{\alpha}}, \\
\bar{u}_{\alpha}(t)=e^{-\underline{\mu}_{\alpha} t}\left(\bar{\omega}_{\alpha}-\frac{\underline{\rho}_{\alpha}}{\underline{\mu}_{\alpha}}\right)+\frac{\underline{\rho}_{\alpha}}{\underline{\mu}_{\alpha}}, \\
{[u(t)]^{\alpha}=\left[\underline{u}_{\alpha}(t), \bar{u}_{\alpha}(t)\right] .}
\end{gathered}
$$

Example 2.2 Consider the fuzzy initial value problem

$$
\begin{aligned}
& u^{\prime}(t)+[-1]^{\alpha} u(t)=[2]^{\alpha}, t>0 \\
& u(0)=[1]^{\alpha}
\end{aligned}
$$

using the fuzzy Laplace transform, where

$[-1]^{\alpha}=[-2+\alpha,-\alpha],[1]^{\alpha}=[\alpha, 2-\alpha],[2]^{\alpha}=[1+\alpha, 3-\alpha]$. 
If $u$ is (1)-differentiable, (1) solution is

$$
\begin{gathered}
\underline{u}_{\alpha}(t)=\left(\frac{(1+\alpha)+(2-\alpha)^{2}}{\sqrt{\alpha(2-\alpha)}}\right) \sin (\sqrt{\alpha(2-\alpha)} t)+\alpha \cos (\sqrt{\alpha(2-\alpha)} t) \\
+\left(\frac{3-\alpha}{\alpha}\right)\left(\frac{e^{\sqrt{\alpha(2-\alpha)} t}+e^{-\sqrt{\alpha(2-\alpha)} t}}{2}-1\right) \\
\bar{u}_{\alpha}(t)=\left(\frac{(3-\alpha)+\alpha^{2}}{\sqrt{\alpha(2-\alpha)}}\right) \sin (\sqrt{\alpha(2-\alpha)} t)+(2-\alpha) \alpha \cos (\sqrt{\alpha(2-\alpha)} t) \\
+\left(\frac{1+\alpha}{2-\alpha}\right)\left(\frac{e^{\sqrt{\alpha(2-\alpha)} t}+e^{-\sqrt{\alpha(2-\alpha)} t}}{2}-1\right) \\
{[u(t)]^{\alpha}=\left[\underline{u}_{\alpha}(t), \bar{u}_{\alpha}(t)\right] .}
\end{gathered}
$$

If $u$ is (2)-differentiable, (2) solution is

$$
\begin{gathered}
\underline{u}_{\alpha}(t)=e^{\alpha t}\left(\frac{\alpha^{2}+3-\alpha}{\alpha}\right)-\left(\frac{3-\alpha}{\alpha}\right), \\
\bar{u}_{\alpha}(t)=e^{(2-\alpha) t}\left(\frac{(2-\alpha)^{2}+1+\alpha}{2-\alpha}\right)-\left(\frac{1+\alpha}{2-\alpha}\right), \\
{[u(t)]^{\alpha}=\left[\underline{u}_{\alpha}(t), \bar{u}_{\alpha}(t)\right] .}
\end{gathered}
$$

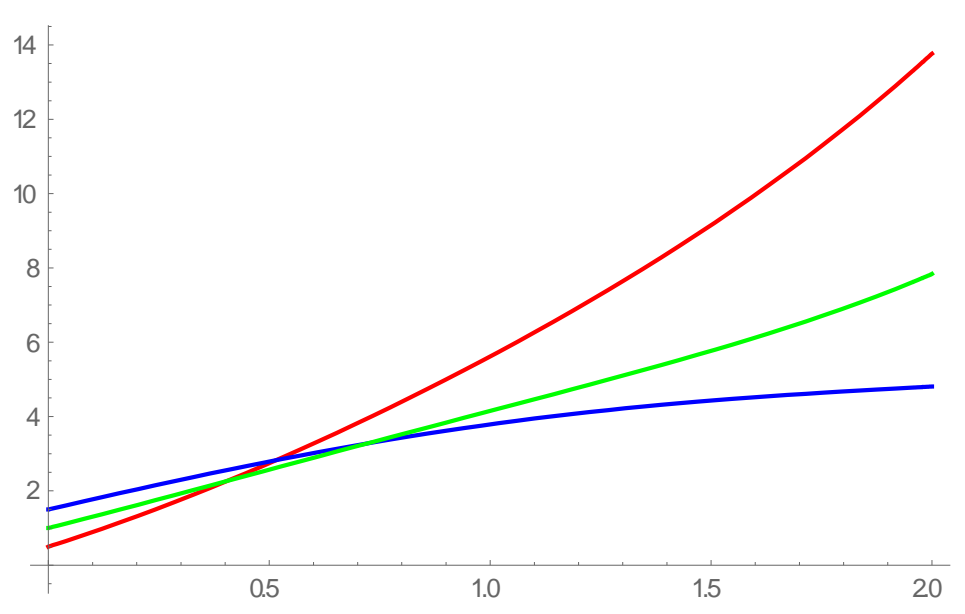

Figure 3 (1) solution for $\alpha=0.5$ 


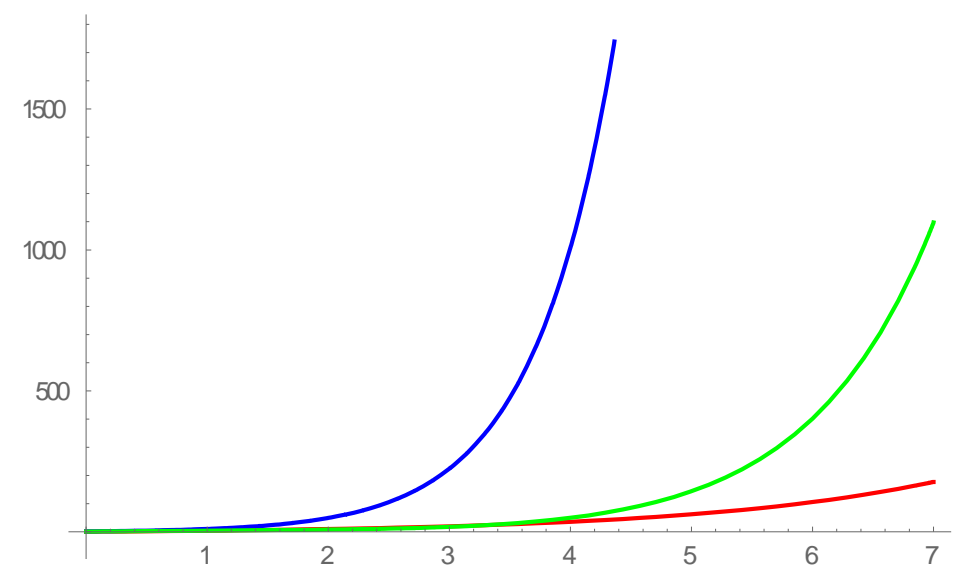

Figure 4 (2) solution for $\alpha=0.5$

$$
\text { red } \rightarrow \underline{u}_{\alpha}(t), \text { blue } \rightarrow \bar{u}_{\alpha}(t), \text { green } \rightarrow u(t)
$$

From Definition 1.3 and since $[u(x)]^{\alpha}$ is positive fuzzy function, according to figure 3, (1) solution is a valid fuzzy number for $x \in[0,0.514863]$, according to figure 4 , (2) solution $\mathrm{i}$

$\mathrm{s}$ a valid fuzzy number for $t>0$.

\section{CONCLUSIONS}

In this study, solutions of fuzzy initial value problems for first-order fuzzy differential equations with positive and negative fuzzy number coefficients are investigated by fuzzy Laplace transform. Generalized differentiability is used. Examples are solved and the figures of the solutions are drawn. It is shown that whether the solutions are valid fuzzy functions or not. Also, it is shown that solutions are valid fuzzy functions in different intervals for each $\alpha$-cut.

\section{REFERENCES}

[1] Kadel A. and Byatt W.J., Fuzzy Differential Equations, in: Proceedings of International Conference Cybernetics and Society, Tokyo, (1978) 1213-1216.

[2] Zadeh L.A., The Concept of a Linguistic Variable and its Application to Approximate Reasoning, Information Sciences, 8 (1975) 199-251.

[3] Hüllermeier E., An Approach to Modelling and Simulation of Uncertain Dynamical Systems, International Journal of Uncertainty, Fuzziness and Knowledge-Based Systems, 5-2 (1997) 117-137.

[4] Kaleva O., Fuzzy Differential Equations, Fuzzy Sets and Systems, 24-3 (1987) 301-317.

[5] Gültekin H. and Altınışık N., On Solution of Two-Point Fuzzy Boundary Value Problems, The Bulletin of Society for Mathematical Services and Standards, 11 (2014) 31-39.

[6] Bede B., Rudas I.J. and Bencsik A.L., First Order Linear Fuzzy Differential Equations under Generalized Differentiability, Information Sciences, $177-7$ (2007) 1648-1662.

[7] Gültekin Çitil H., The Relationship between the Solutions according to the Noniterative Method and the Generalized Differentiability of the Fuzzy Boundary Value Problem, Malaya Journal of Matematik, 6-4 (2018) 781-787.

[8] Ceylan T. and Altınışık N., Fuzzy Eigenvalue Problem with Eigenvalue Parameter Contained in the Boundary Condition, Journal of Science and Arts, 3-44 (2018) 589-602. 
[9] Allahviranloo T. and Barkhordari Ahmadi M., Fuzzy Laplace Transforms, Soft Computing, 14-3 (2010) 235-243.

[10] Patel K.R. and Desai N.B., Solution of Fuzzy Initial Value Problems by Fuzzy Laplace Transform, Kalpa Publications in Computing, 2 (2017) 25-37.

[11] Patel K.R. and Desai N.B., Solution of Variable Coefficient Fuzzy Differential Equations by Fuzzy Laplace Transform, International Journal on Recent and Innovation Trends in Computing and Communication, 5-6 (2017) 927-942.

[12] Liu H.-K., Comparison Results of Two-Point Fuzzy Boundary Value Problems, International Journal of Computational and Mathematical Sciences, 5-1 (2011) 1-7.

[13] Salahshour S. and Allahviranloo T., Applications of Fuzzy Laplace Transforms, Soft Computing, 17-1 (2013) 145-158.

[14] Shirin S. and Saha G.K., A New Computational Methodology to Find Appropriate Solutions of Fuzzy Equations, Mathematical Theory and Modeling, 2-1 (2011) 1-10.

[15] Lakshmikantham V. and Mohapatra R.N., Theory of Fuzzy Differential Equations and Inclusions, Taylor and Francis, London, New York, 2003.

[16] Khastan A. and Nieto J.J., A Boundary Value Problem for Second Order Fuzzy Differential Equations, Nonlinear Analysis: Theory, Methods and Applications, 72-9-10 (2010) 3583-3593. 